\title{
A potential role of karyopherin a2 in the impaired maturation of dendritic cells observed in glioblastoma patients
}

\author{
Konstantinos Gousias ${ }^{1,2}$, Alexander von Ruecker ${ }^{3}$, Gerrit H. Gielen ${ }^{4}$, Pitt Niehusmann ${ }^{4}$, Andreas Waha ${ }^{4}$, \\ Hartmut Vatter ${ }^{1}$, Matthias Simon ${ }^{1}$ \\ ${ }^{1}$ Department of Neurosurgery, University Hospital of Bonn, Sigmund-Freud-Strasse 25, 53105 Bonn, Germany. \\ ${ }^{2}$ Department of Neurosurgery, University Hospital of Bochum, Bergmannsheil, Bürkle-de-la-Camp-Platz 1, 44789 Bochum, \\ Germany \\ ${ }^{3}$ Department of Pathology, University Hospital of Bonn, Sigmund-Freud-Strasse 25, 53105 Bonn, Germany. \\ ${ }^{4}$ Department of Neuropathology, University Hospital of Bonn, Sigmund-Freud-Strasse 25, 53105 Bonn, Germany.
}

\section{A B S T R A C T}

\begin{abstract}
Aim: Patients with glioblastomas demonstrate well-documented immunological impairments including decreased numbers of mature dendritic cells (DCs). Recent data identified karyopherin a2 (KPNA2), a nucleocytoplasmic shuttling receptor, as diagnostic and prognostic biomarker for gliomas. The aim of this ongoing study is to correlate parameters of immunity and nucleocytoplasmic transport in glioblastoma patients. Methods: We preoperatively collected serum from 17 patients with glioblastomas and determined DC subsets (HLA DR+ Lin-, CD34-, CD45+, CD123+, CD11+ were analyzed) using a 6-color flow cytometry panel. Expression levels of KPNA2 and nuclear accumulation of p53 were evaluated semi-quantitatively by immunohistochemistry. $0^{6}$-methylguanine DNA methyltransferase (MGMT) and isocitrate dehydrogenase-1 (IDH-1) status were assessed by pyrosequencing and immunohistochemistry, respectively. Results: Median expression levels for both KPNA2 and p53 were 5-10\%. IDH-1-R132H mutation and MGMT promoter hypermethylation was detected in $3 / 16$ and $1 / 9$ patients, respectively. Mean counts of total mature DCs, myeloid DCs and plasmacytoid DCs were 9.6, 2.1, 3.4 cells/ $\mu \mathrm{L}$. A preliminary analysis suggests an association between low KPNA2 nuclear expression and increased numbers of mature DCs. However, this correlation did not reach statistical significance so far $(P=0.077)$. Conclusion: Our preliminary data may indicate a role of KPNA2 in the impaired maturation of DCs observed in glioblastoma patients.
\end{abstract}

Key words: Glioblastomas, isocitrate dehydrogenase-1, karyopherin a2, mature dendritic cells, $0^{6}$-methylguanine DNA methyltransferase, p53

\section{INTRODUCTION}

Patients with glioblastomas demonstrate well-documented impairments of their immune system, including reduced values of mature dendritic cells (DCs). ${ }^{[1-4]}$ DCs is the most potent antigen-presenting cell population and therefore key regulators of adaptive immunity. Apart from their defense against infectious diseases they may also mediate antitumor responses. ${ }^{[5,6]}$ Their maturation/differentiation plays a pivotal role in their function. After recognizing and capturing

\begin{tabular}{|l|l|}
\hline \multicolumn{2}{|c|}{ Access this article online } \\
\hline Quick Response Code: & \\
\hline & Website: \\
\hline & www.nnjournal.net \\
& \\
\hline
\end{tabular}

antigens/tumor cells they undergo maturation, i.e., they upregulate major histocompatibility complex class II and co-stimulatory molecules at cell surface, they migrate to $\mathrm{T}$ cell rich zones and secrete cytokines to induce an antigen-specific $\mathrm{T}$ cell response. ${ }^{\left[{ }^{7]}\right.}$

DCs maturation may be mediated by known signaling pathways/transcription factors, for instance, nuclear factor- $\kappa \mathrm{B}(\mathrm{NF}-\kappa \mathrm{B})$, which is triggered after activation of the toll-like receptors (TLRs) by invading microorganisms. ${ }^{[7]}$ Once in the nucleus, NF- $\mathrm{KB}$ may induce the transcription of various genes involving in immune and inflammatory responses. ${ }^{[8]}$ Nuclear import of NF-KB is mediated by the karyopherin a2/importin unit 2 complex (KPNA2), a well-studied member of the family of karyopherins. ${ }^{[9]}$

Karyopherins are nucleocytoplasmatic shuttling receptors and comprise importins and exportins. They

Corresponding Author: Dr. Konstantinos Gousias, Department of Neurosurgery, University Hospital of Bonn,

Sigmund-Freud-Strasse 25, 53105 Bonn, Germany. E-mail: kostasgousias@yahoo.com 
have been linked to signal transduction pathways and cell cycle control mechanisms. ${ }^{[10-13]}$ KPNA2 mediates the nuclear import of large molecules $(>40 \mathrm{kDa}$, most of the proteins and RNAs) by binding to a specific recognition sequence called the nuclear localization signal (NLS). After entering the nucleus, the NLS-containing macromolecule is dissociated by RanGTP and KPNA2 recycles back to the cytoplasm.

Recent data suggests a role for the nucleocytoplasmic transport, in particular for KPNA2, in gliomagenesis. We have previously identified low expression of KPNA2 as an independent prognostic factor for better overall survival (OS) and progression-free survival (PFS) in patients with infiltrative gliomas. ${ }^{[14,15]}$ KPNA2 has been also recognized as a prognostic factor in patients with meningiomas ${ }^{[16]}$ as well as in patients with other solid tumors. ${ }^{[17-20]}$

The aim of our work is to investigate the role of nucleocytoplasmic import and of other known biomarkers in the maturation procedure of DCs. In a recent work, we analyzed the preoperative phenotype of DCs in patients with gliomas. ${ }^{[4]}$ In the present ongoing study we determined also parameters of nucleocytoplasmic import (KPNA2) as well as other glioma-associated molecular markers such as $O^{6}$-methylguanine DNA methyltransferase (MGMT) promoter hypermethylation, isocitrate dehydrogenase-1-R132H (IDH-1-R132H) gene mutation status and nuclear accumulation of p53 within the tissue specimens and analyzed a putative correlation between them. ${ }^{[21-23]}$ Our preliminary results imply a possible role of KPNA2 in the known impaired maturation of DCs in patients with glioblastomas.

\section{METHODS}

\section{Patients and clinical characteristics}

We analyzed preoperatively collected fasting morning serum from 17 consecutive adult (median age: 54 years, range: $33-78$ years; $58.8 \%$ male) patients with subsequently histologically confirmed de novo glioblastomas operated at the Department of Neurosurgery of the University Hospital of Bonn between November of 2010 and February of 2011 for DC subpopulations. In addition, surgical specimens from our patients were analyzed for expression of KPNA2 $(n=16)$ and nuclear accumulation p53 $(n=17)$ as well as for IDH-1-R132H mutation status $(n=16)$ and MGMT promoter hypermethylation $(n=9)$. Nonneoplastic brain tissues from two patients who underwent surgery for epilepsy served as controls. The circadian rhythm of the immunological parameters and the possible effect of dexamethasone administration were considered at the blood collection as previously described. ${ }^{[4]}$
All tumors were located in the supratentorial compartment. Three (17.6\%) patients had a diagnostic biopsy only due to the eloquent location of the tumor and $35.3 \%$ of resections were gross total. All patients underwent chemo- and radiotherapy after surgery. The demographics of our study population are shown in Table 1. Patients with a history of previous brain tumor or other cancer, radio- or chemotherapy or of an immunological or hematological disease were excluded from the study. The patients' samples were collected after their informed consents were obtained in accordance with the tenets of the declaration of Helsinki and approval of the study by the Ethics Committee of the Medical Faculty of the University of Bonn.

\section{Flow cytometry}

Dendritic cell and T-lymphocyte subpopulations values were determined by flow cytometry using six different fluorochromes: fluorescein isothiocyanate (FITC), phycoerythrin (PE), peridininchlorophyllprotein (PerCP), allophycocyanin (APC), PE-Cy7 (PE-Cy7) and APC-Cy7. The following surface and intracellular anti-human monoclonal antibodies were used: CD45-APC Cy7 (clone 2D1), CD4-PE (clone RPA-T4), CD3-PerCP (clone SK7), HLA-DR-PerCP (clone L243), CD11c-APC clone (S-HCL-3), lineage-FITC (lin-1 cocktail), CD34-FITC (clone 8G12), CD123-PE-Cy7 (clone 6H6, ebioscience, San Diego, CA) as well as isotype controls.

Cells were surface stained according to the manufacturers' protocols. DCs were isolated as previously described. ${ }^{[4]}$ Briefly, DCs were gated as HLA-DR (MCH class II) positive, lineage-negative, CD34 negative, and CD45 positive (HLA DR+/lin-/CD34-/CD45+). DCs were further subclassified as myeloid DCs (mDCs) or plasmacytoid DCs (pDCs) based on their reciprocal

\begin{tabular}{lc}
\hline Table I: Patient demographics and tumor characteristics \\
\hline Variable & Absolute numbers (\%) \\
\hline Number of patients & 17 \\
Median age (range) & $54(33-78)$ years \\
Males & $10(58.8)$ \\
Maximum tumor diameter* $\leq 3 \mathrm{~cm}$ & $7(41.1)$ \\
Resection** & \\
GTR & $6(35.3)$ \\
STR & $8(47.0)$ \\
Biopsy & $3(17.6)$ \\
Preoperative KPS: $90-100 \%$ & $12(70.5)$ \\
Postoperative KPS: $90-100 \%$ & $11(64.7)$ \\
Preoperative seizures: yes & $6(35.3)$ \\
Eloquence**: Yes & $10(58.8)$ \\
Radiotherapy: Yes & $17(100)$ \\
Chemotherapy: Yes & $17(100)$ \\
\hline
\end{tabular}

${ }^{*}$ Maximum tumor diameter has been defined as the longest (any) diameter of contrast enhancing mass area in postcontrast T1-weighted MRI datasets; ** Extent of resection was classified according to the postoperative MRI (2-3 days after surgery); ${ }^{* * *}$ Tumors were categorized as eloquent if they were growing into the primary sensorimotor or visual cortex, Broca's or Wernicke's area/the dominant angular gyrus area, the basal ganglia, thalamus or internal capsule. MRI: magnetic resonance imaging; KPS: karnofsky performance score 


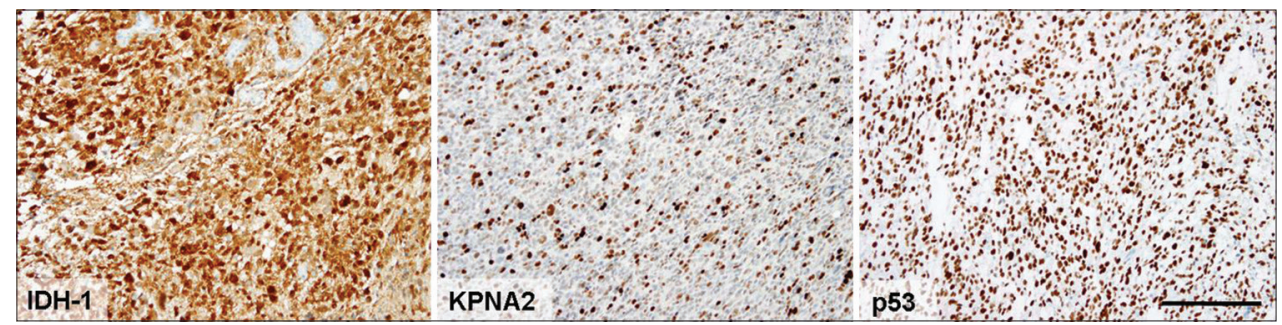

Figure 1: Exemplary images of immunohistochemical evaluation of isocitrate dehydrogenase (IDH-1 [R132H]) and karyopherin a2 (KPNA2) expression as well as nuclear accumulation of p53 protein. Immunohistochemical staining with antibodies against mutated IDH-1 (R132H) shows a strong cytoplasmic immunoreactivity in the tumor cells. Nuclear expression of KPNA2 is detected in a subpopulation of tumor cells. Nuclear p53 accumulation can be observed in $>50 \%$ of the tumor

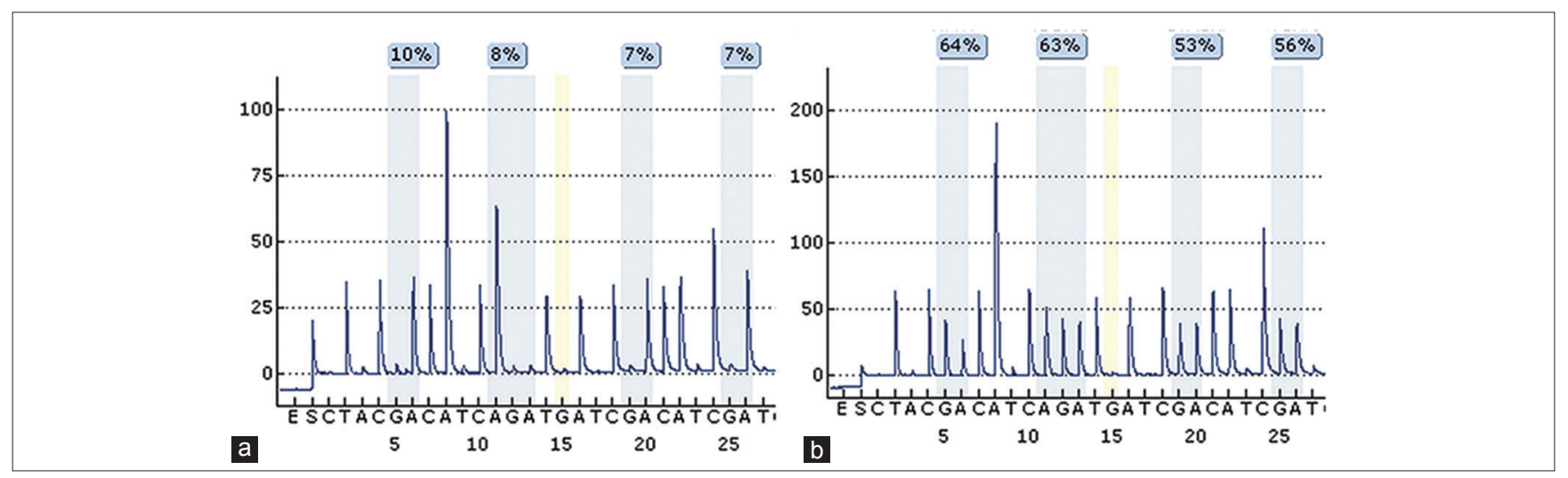

Figure 2: Pyrosequencing was used for quantitative analysis of $O^{6}$-methylguanine DNA methyltransferase promoter methylation. Pyrogram demonstrating (a) an unmethylated and (b) methylated glioblastoma tissue. Each colored box includes one of the four studied CpG positions (CpGs 9-12). The incorporation of the bases guanine and adenine represent the methylated and unmethlylated fractions, respectively. The percentages given in both pyrograms reflect the methylated fractions (fractions over $10 \%$ define a methylated sample)

expression of CD11c (a-integrin) and CD123 (IL-3 receptor a), respectively. Figures 1 and 2 of our previous publication illustrate the classification/gating steps. ${ }^{[4]}$ HLA-DR, CD11c, CD45, CD123 are co-stimulatory surface molecules, which have been identified in the relevant literature as markers of maturation. ${ }^{[24-27]}$ CD34 is a marker for all stem cells and the proportion of precursor DCs. ${ }^{[28]}$ Therefore, HLA DR+/Lin-/CD34-/CD45 + were defined as total mature DCs. HLA DR +/Lin-/CD34-/CD45+/CD123-/ CD11c- represent less mature DCs, whereas HLA DR+/ Lin-/CD34-/CD45+/CD11c+/CD123- mDCs and HLA DR+/Lin-/CD34-/CD45+/CD123+/CD11c- pDCs mature DCs in advanced stages of maturation.

\section{Immunohistochemistry}

Neuropathological analysis of glioblastomas comprised hematoxylin/eosin staining as well as immunohistochemistry with monoclonal antibodies directed against the microtubule-associated protein 2 (MAP2, Sigma, Steinheim, Germany), polyclonal antibodies directed against glial fibrillary acid protein (GFAP, Sigma, Steinheim, Germany) and monoclonal antibodies directed against Ki67 (MIB1, Dako, Glastrop, Denmark).

IDH-1-R132H mutation status (monoclonal mouse antibody H09 directed against mutated IDH-1 R132H mutation, Dianova, Hamburg, Germany) and KPNA2 immunoreactivity (goat polyclonal SC6917; Santa Cruz Biotechnology, Santa Cruz, USA; dilution 1:100) were assessed and visually scored independently by two experienced neuropathologists (PN, GHG) as previously described [Figure 1]. ${ }^{[14]}$ Immunohistochemical staining with a monoclonal antibody against p53 (clone DO-7, Dako, Glostrup, Denmark), in a dilution of 1:150, were performed on the Ventana Immunostainer (Roche, Mannheim, Germany), with a closed avidin-biotin complex Ventana Detection System (Ventana). Positive and negative controls were also performed using glioblastoma tissue with p53 overexpression [Figure 1]. Tumors were assigned to immunoreactivity classes of KPNA2 and p53 based on the percentage of moderately or strongly immunopositive cell nuclei $(<1 \%, 1 \%-<5 \%$, $5 \%-<10 \%, 10 \%-<20 \%, 20 \%-<50 \%$ and $\geq 50 \%)$.

\section{Pyrosequencing}

The quantitative analysis of MGMT promoter methylation by pyrosequencing was performed as previously described. ${ }^{[29]}$ Briefly, the first four CpG sites are assayed for a primer extension reaction. Methylated fractions $>10 \%$ at all positions define a methylated sample [Figure 2].

\section{Statistical analysis}

Statistical analyses of the data were performed using commercially available software (SSPS 21.0, IBM Deutschland, Ehningen, Germany). Comparisons of samples were performed using standard methods (Pearson's Chi-square, Fisher's exact test). $P<0.05$ (two-tailed) were considered to be statistically 
significant. Cut-off values for nonparametric statistics were set at the median of each variable, that is, the studied subgroups were as following KPNA2: $<5 \%$ vs. $\geq$ $5 \%$ positive cell nuclei; p53: $<5 \%$ vs. $\geq 5 \%$ positive cell nuclei; total mature DC: $<5.9$ vs. $\geq 5.9$ cells/ $\mu \mathrm{L} ; \mathrm{pDC}:<$ 1.6 vs. $\geq 1.6$ cells $/ \mu \mathrm{L} ; \mathrm{mDC}:<0.6$ vs. $\geq 0.6$ cells $/ \mu \mathrm{L}$; age: $<54$ vs. $\geq 54$; preoperative and postoperative Karnofsky performance index (KPI): < 90 vs. $\geq 90$.

\section{RESULTS}

The relative proportions of total mature DCs as well as subsets of mature DCs in patients with glioblastomas were as following: total DCs as proportion of WBC: $0.11 \%$ (0.05-0.16\%), pDC CD123+ CD11c- as proportion of total DCs: $28.3 \%$ (16.5-40.1\%), mDC CD123- CD11c + as proportion of total DC: $15.5 \%$ (6.0$25.1 \%)$, CD123-CD11C- as proportion of total DCs: $54.5 \%$ (38.7-70.3\%). Similarly, counts (mean, 95\% CI, median) (cells $/ \mu \mathrm{L}$ ) of mature DCs were: total mature DCs: 9.6 (4.3-14.9), 5.9, pDC CD123+ CD11c-: 3.4 (1.1-5.8), 1.6, mDC CD123- CD11c+: 2.1 (0.6-3.6), 0.6 [Table 2].

Median expression levels for both KPNA2 and p53 were 5-10\% [Figure 3]. IDH-1-R132H mutations were detected in 3/16 patients. All glioblastoma patients with mutant IDH-R132H experienced a sudden onset ( $<3$ months) of their symptoms, which imply that these patients harbored primary rather than secondary glioblastomas. Representative results of KPNA-, p53- and IDH-1-immunohistochemistry are shown in Figure 1. MGMT promoter hypermethylation was observed in 1/9 tumors [Figure 2].

A preliminary analysis suggests an association between lower KPNA2 nuclear expression and increased numbers of mature DC. All patients with low KPNA2 ( $<5 \%$ ) expression and only 33.3\% of patients with KPNA2 $\geq 5 \%$ demonstrated counts of mature DCs over the median ( $\geq 5.9$ cells $/ \mu \mathrm{L}$ ). However, this correlation did not reach statistical significance so far (total mature DC $\geq 5.9$ cells/ $\mu \mathrm{L}$ : KPNA2 < 5\% vs. $\geq 5 \% / 100 \%$ vs. $33.3 \%$, Pearson's Chi-square: $P=0.038$, Fisher's exact test: $P=0.077$, both two-sided; Fisher's exact test is most appropriate, since study population is limited) [Figure 4].

A trend between KPNA2 expression and IDH-R132H mutation status has been observed. Patients expressing lower KPNA2 exhibit also frequently mutant IDH-1-R132H (mutant IDH-1-R132H: KPNA2 < 5\% vs. $\geq 5 \% / 66.7 \%$ vs. 7.7\%, Pearson's Chi-square: $P=0.018$, Fisher's exact test: $P=0.071$, both two-sided) [Figure 4]. No mutant IDH-1-R132H status was seen in patients with KPNA2 $\geq 10 \%$. No other significant correlations between expressions of KPNA2, p53, MGMT promoter hypermethylation and IDH-1-R132H mutation status have been observed.

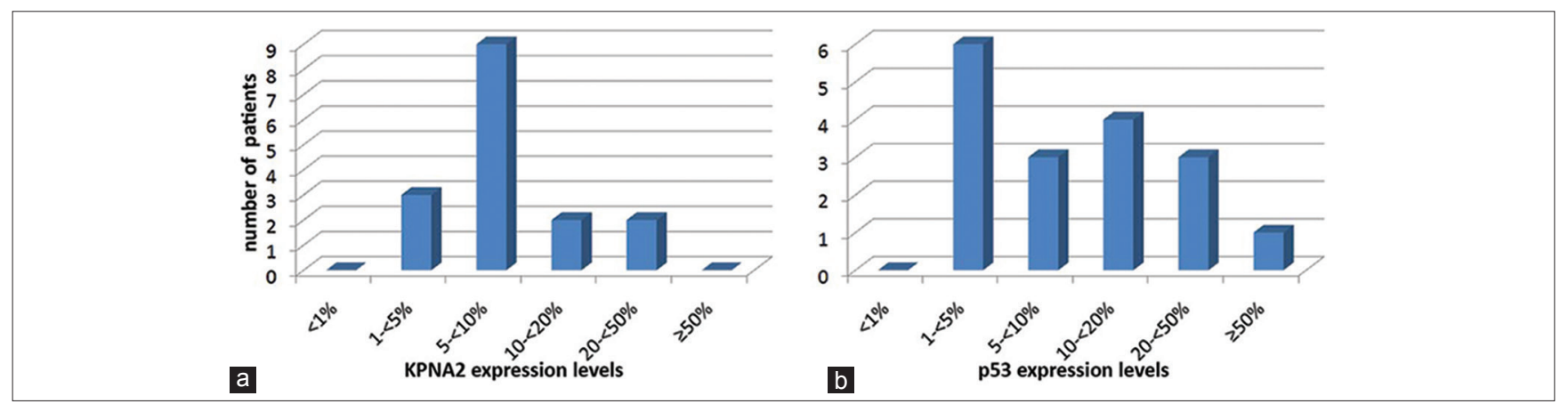

Figure 3: Frequency distribution bar diagram illustrating the nuclear expression levels of (a) karyopherin a2 (KPNA2) and (b) p53. KPNA2 and p53 expression were analyzed for nonparametric statistics as dichotomized variables with their respective median $(5 \%-<10 \%$ for both variables) being set as cut off value. Thus, low KPNA2 and low p53 expression were defined as expression levels $<5 \%$
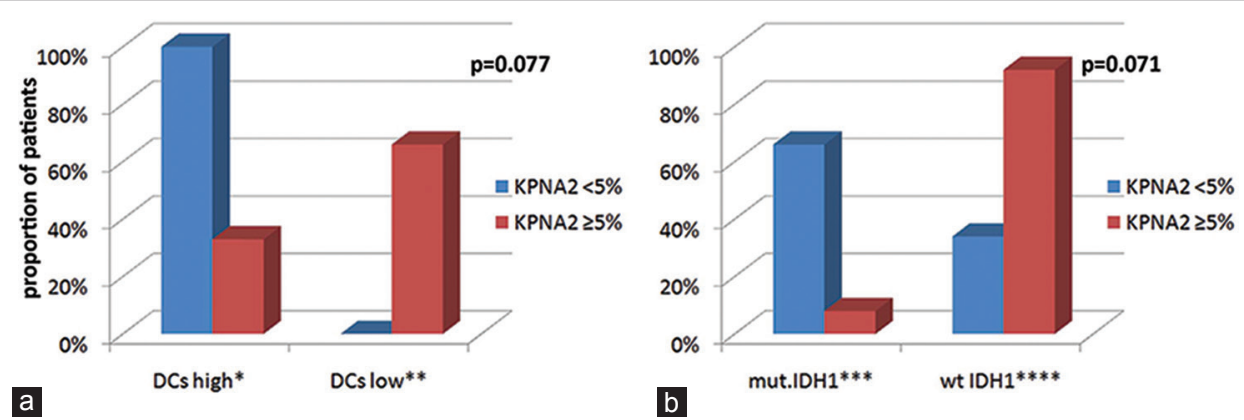

Figure 4: Correlations bar diagram. The y axis shows the relative proportion of our cohort with different karyopherin a2 (KPNA2) expression demonstrating (a)

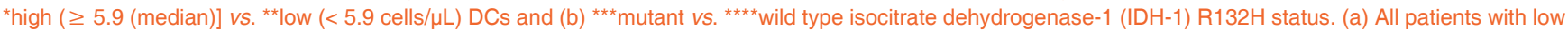
KPNA2 $(<5 \%)$ compared to only $33.3 \%$ of the patients with KPNA2 $\geq 5 \%$ demonstrated DCs high $(P=0.077)$. (b) Mutant IDH-1 status was seen more frequently in patients with low $(<5 \%)$ than in those with KPNA2 $\geq 5 \%(66.7 \%$ vs. only $7.7 \%, P=0.071)$ 
No significant imbalances between subgroups of KPNA2 and p53 expression as well as MGMT promoter methylation and IDH-1 mutation status (see statistical analysis) with respect to age, gender, type of resection (resection vs. biopsy), preoperative and postoperative KPI, tumor volume, presence of epilepsy (yes/no), neurological deficits (yes/no) and eloquence of the tumor location (yes/no) were seen [Table 3].

\section{DISCUSSION}

Patients with glioblastomas exhibit well-documented immunological abnormalities; in particular, they demonstrate an impaired cellular immunity; that is, reduced counts of effector $\mathrm{T}$ helper and relative accumulation of suppressive $\mathrm{T}$ regulatory cells. ${ }^{[2,4]}$ The APC function of DCs, as reflected by the ability to stimulate allogeneic $\mathrm{T}$ cells, is also altered/ diminished. ${ }^{[30]}$ Patients with glioblastomas demonstrate also reduced values of mature DCs compared to patients with gliomas WHO grade I-III or healthy donors. ${ }^{[4]}$

The maturation of DCs is the key regulator of their APC function, comprises several stages (stem cells/precursors/ poorly differentiated/highly differentiated not activated/ highly differentiated and activated/apoptosis) and includes the up regulation in the nucleus of $\mathrm{MCH}$ class II and specific co-stimulatory molecules, such as CD11c, CD45, CD83, CD86 and CD123..$^{[7,24-27]}$ This up regulation may be generally triggered by transcription factors/stimuli (macromolecules $>40 \mathrm{kDa}$ ), which are being actively translocated from the cell surface (that is, side of "danger signal" production upon recognition of invading microorganisms/antigens) into the nucleus. ${ }^{[7,8]}$ Consequently, molecules involved in the nuclear import of these transcription factors, such as karyopherins, may also affect the maturation procedures.

Recognition receptors of immature DCs, such as TLRs, may be triggered by invading microorganisms or endogenous inflammatory signals and on their turn they may activate signaling pathways/transcription factors, such as NF- $\kappa \mathrm{B}$, in order to foster their maturation. This pathway is among others characterized by the proteolytic processing of NF- $\mathrm{\kappa B}$ p100 protein to p52 and the translocation of the latter in the nucleus. ${ }^{[31,32]}$ Lind et al. ${ }^{\left[{ }^{[31]}\right.}$ studied NF-אB pathway in vivo in alymphoplasia (Aly) and wild type (WT) mice. Aly mouse expresses mutant molecules that prohibit the induction of NF- $\mathrm{\kappa B}$ pathway and demonstrate an impaired cross-presentation of antigens. Aly failed to translocate p52 to the nucleus after activation with CD40, whereas a normal nuclear p52 translocation occurs in WT. ${ }^{[31]}$ The nuclear import of p52 is mediated by members of karyopherin family proteins (a1-a5, a7). ${ }^{[9,32]}$

Karyopherins are nuclear proteins involved in nucleocytoplasmatic shuttling and have been linked to tumorigenesis. KPNA2 has been identified as a regulator of DNA repair proteins and an activator of apoptosis pathways. ${ }^{[10-13]}$ Recent data suggested a role for KPNA2, also in gliomagenesis. We have recognized in two recent works about KPNA2 as an independent prognostic factor for OS and PFS in 94 patients with infiltrative astrocytomas WHO grade II-IV as well as in 72 patients with anaplastic gliomas (astrocytomas, oligoastrocytomas and oligodendrogliomas WHO grade III). ${ }^{[14,15]}$ KPNA2 has been also identified as a prognostic factor in patients with meningiomas ${ }^{[16]}$ as well as in patients with other solid tumors. ${ }^{[17-20]}$

\begin{tabular}{lcc}
\hline Table 2: Relative proportions and counts of T-lymphocytes, T-helper lymphocytes and DC subsets in our series \\
\hline Immunological parameter & Relative proportion mean $\mathbf{( 9 5 \% ~ C l )}$ & Counts (cells/ $\mathbf{\mu L}) \mathbf{m e a n}(\mathbf{9 5 \%} \mathbf{C l})$, median \\
\hline T cell lymphocytes CD3+ \%WBC* & $6.58(3.24-9.92)$ & $554(348-760), 488$ \\
T helper lymphocytes CD4+ \%WBC & $4.16(1.90-6.42)$ & $348(199-497), 225$ \\
DCs HLA DR+/CD34-/CD45+ \%WBC & $0.11(0.05-0.16)$ & $9.6(4.3-14.9), 5.9$ \\
pDCs CD123+ CD11C- \%DC** & $28.3(16.5-40.1)$ & $3.4(1.1-5.8), 1.6$ \\
mDCs CD123-, CD11C+ \%DC & $15.5(6.0-25.1)$ & $2.1(0.6-3.6), 0.6$ \\
CD123-, CD11C- \%DC & $54.5(38.7-70.3)$ & $10.1(0.4-21.4), 2.1$ \\
\hline
\end{tabular}

*\% WBC: relative proportion of WBCs; **\% DC: relative proportion of DCs. DCs: dendritic cells; WBC: white blood cell; Cl: confidence interval; HLA: human leukocyte antigen; DR: diabetic retinopathy

\begin{tabular}{|c|c|c|}
\hline Variable & p53 $<5 \%$ vs. $\geq 5 \%, P$ & KPNA2 $<5 \%$ vs. $\geq 5 \%, P$ \\
\hline Males & $57.1 \%$ vs. $60.0 \%$, n.s* & $66.7 \%$ vs. $53.8 \%$, n.s \\
\hline Max tumor diameter $\leq 3 \mathrm{~cm}$ & $71.4 \%$ vs. $25.0 \%$, n.s & $33.3 \%$ vs. $54.5 \%$, n.s \\
\hline \multicolumn{3}{|l|}{ Resection } \\
\hline Cytoreductive surgery & $57.1 \%$ vs. $88.9 \%$, n.s & $66.7 \%$ vs. $83.3 \%$, n.s \\
\hline Diagnostic biopsy & $42.9 \%$ vs. $11.1 \%$, n.s & $33.3 \%$ vs. $16.7 \%$, n.s \\
\hline Preoperative KPS: $90-100 \%$ & $57.1 \%$ vs. $22.2 \%$, n.s & $66.7 \%$ vs. $33.3 \%$, n.s \\
\hline Postoperative KPS: $90-100 \%$ & $42.9 \%$ vs. $30.0 \%$, n.s & $66.7 \%$ vs. $30.8 \%$, n.s \\
\hline Preoperative seizures: yes & $42.9 \%$ vs. $33.3 \%$, n.s & $66.7 \%$ vs. $25.0 \%$, n.s \\
\hline Eloquence: yes & $57.1 \%$ vs. $66.7 \%$, n.s & $66.7 \%$ vs. $58.3 \%$, n.s \\
\hline
\end{tabular}

${ }^{*}$.s: no significant; $P>0.05$. KPNA2: karyopherin a2; KPS: karnofsky performance score 
In the present ongoing study, we determined and correlated DC subpopulations as well as expression of KPNA2 in patients with glioblastomas. Since KPNA2 is thought to mediate the nuclear import of certain transcription factors, which may induce the maturation of DCs, a certain correlation has been expected. Indeed, our preliminary analysis suggests an association between low KPNA2 nuclear expression and increased numbers of mature DCs. However, this correlation did not reach statistical significance so far (Fisher exact test, $P=0.077$ ) probably due to the limited studied population. The observed inverse correlation between KPNA2 expression and counts of mature DCs is not surprising, since higher KPNA2 expression ${ }^{[14]}$ and decreased counts of mature $\mathrm{DCs}^{[4]}$ have been both found to characterize patients with malignant gliomas, The idea of the possible role of KPNA2 also in the regulation of the immunity of glioblastoma patients is tempting; in such a case an additional therapeutic target for the immunotherapy may have been identified.

To our best knowledge, this is the first study focusing on the role of importins in the maturation of DCs. Some evidence of a role of karyopherins (only exportins) in the function of DCs has been previously elucidated. ${ }^{[33]}$ Chemnitz et al. ${ }^{[33]}$ studied in vitro the role of the exportin chromosome region maintenance protein $1 /$ exportin 1(CRM1) in the maturation and activation of DCs. Inhibition of CRM1 by Leptomycin B down regulated the expression of the co-stimulatory molecule CD83 and abrogated the ability of allogeneic $\mathrm{T}$ cell stimulation. ${ }^{[33]}$

Established prognostic molecular biomarkers, such as MGMT promoter methylation and IDH-1 mutation status were also included in our analysis. Patients with low KPNA2 expression exhibit frequently (not statistically significant) mutant IDH-1-R132H (Fisher's exact test: $P=0.071$ ). The clinical history of the patients with mutant IDH-1-R132H status (sudden onset of symptoms $<3$ months) does not suggest a secondary genesis of glioblastomas. However, a possible association between lower KPNA2 expression and genesis of secondary glioblastomas could not be excluded. An inverse correlation of KPNA2 expression and IDH-1 immunostaining in patients with malignant gliomas was found also previously. ${ }^{[14]}$

Furthermore, we tested whether expression of KPNA2 and DC subpopulations correlated with clinical factors, such as gender, age, preoperative and postoperative Karnofsky Index, preoperative presence of seizures or neurological deficits, tumor diameter, tumor eloquence and degree of resection. No significant associations were found.
We presented the preliminary analysis of our ongoing study on the immunity of patients with glioblastomas. Our first results comprise a limited studied population; therefore, far reaching conclusions may not be drawn. However, our data may be taken into consideration in order to design future larger relevant studies, in particular animal models with knock out techniques that may further clarify the role of importins in the maturation of DCs and in general in the immunological abnormalities observed in patients with glioblastomas.

\section{ACKNOWLEDGMENTS}

We thank Alexandra Breuer for her excellent assistance and technical support.

\section{REFERENCES}

1. Zisakis A, Piperi C, Themistocleous MS, Korkolopoulou P, Boviatsis EI, Sakas DE, Patsouris E, Lea RW, Kalofoutis A Comparative analysis of peripheral and localised cytokine secretion in glioblastoma patients. Cytokine 2007;39:99-105.

2. Fecci PE, Mitchell DA, Whitesides JF, Xie W, Friedman AH, Archer GE, Herndon JE 2nd, Bigner DD, Dranoff G, Sampson JH. Increased regulatory $\mathrm{T}$-cell fraction amidst a diminished $\mathrm{CD} 4$ compartment explains cellular immune defects in patients with malignant glioma. Cancer Res 2006;66:3294-302.

3. Gousias K, Markou M, Arzoglou V, Voulgaris S, Vartholomatos G, Kostoula A, Polyzoidis K, Kyritsis AP. Frequent abnormalities of the immune system in gliomas and correlation with the WHO grading system of malignancy. J Neuroimmunol 2010;226:136-42.

4. Gousias K, von Ruecker A, Voulgari P, Simon M. Phenotypical analysis, relation to malignancy and prognostic relevance of ICOS $+\mathrm{T}$ regulatory and dendritic cells in patients with gliomas. J Neuroimmunol 2013;264:84-90.

5. Banchereau J, Briere F, Caux C, Davoust J, Lebecque S, Liu YJ, Pulendran B, Palucka K. Immunobiology of dendritic cells. Annu Rev Immunol 2000;18:767-811.

6. Fong L, Engleman EG. Dendritic cells in cancer immunotherapy. Annu Rev Immunol 2000;18:245-73.

7. Dalod M, Chelbi R, Malissen B, Lawrence T. Dendritic cell maturation: functional specialization through signaling specificity and transcriptional programming. EMBO J 2014;33:1104-16.

8. Pahl HL. Activators and target genes of Rel/NF-kappaB transcription factors. Oncogene 1999;18:6853-66.

9. Cunningham MD, Cleaveland J, Nadler SG. An intracellular targeted NLS peptide inhibitor of karyopherin alpha: NF-kappa B interactions. Biochem Biophys Res Commun 2003;300:403-7.

10. Chen YC, Su YN, Chou PC, Chiang WC, Chang MC, Wang LS, Teng SC, Wu KJ. Overexpression of NBS1 contributes to transformation through the activation of phosphatidylinositol 3-kinase/Akt. J Biol Chem 2005;280:32505-11.

11. Teng SC, Wu KJ, Tseng SF, Wong CW, Kao L. Importin KPNA2, NBS1, DNA repair and tumorigenesis. J Mol Histol 2006;37:293-9.

12. Tseng SF, Chang CY, Wu KJ, Teng SC. Importin KPNA2 is required for proper nuclear localization and multiple functions of NBS1. JBiol Chem 2005;280:39594-600.

13. Zannini L, Lecis D, Lisanti S, Benetti R, Buscemi G, Schneider C, Delia D. Karyopherin-alpha2 protein interacts with Chk2 and contributes to its nuclear import. J Biol Chem 2003;278:42346-51.

14. Gousias K, Becker AJ, Simon M, Niehusmann P. Nuclear karyopherin a2: a novel biomarker for infiltrative astrocytomas. $J$ Neurooncol 2012;109:545-53.

15. Gousias K, Niehusmann P, Gielen G, Simon M, Boström J. 
KPNA2 predicts long term survival in patients with anaplastic oligoastrocytomas. J Clin Neurosci 2014;21:1719-24.

16. Gousias K, Niehusmann P, Gielen GH, Simon M. Karyopherin a2 and chromosome region maintenance protein 1 expression in meningiomas: novel biomarkers for recurrence and malignant progression. J Neurooncol 2014;118:289-96.

17. Zheng M, Tang L, Huang L, Ding H, Liao WT, Zeng MS, Wang HY. Overexpression of karyopherin-2 in epithelial ovarian cancer and correlation with poor prognosis. Obstet Gynecol 2010;116:884-91.

18. Dahl E, Kristiansen G, Gottlob K, Klaman I, Ebner E, Hinzmann B, Hermann K, Pilarsky C, Durst M, Klinkhammer-Schalke M, Blaszyk H, Knuechel R, Hartmann A, Rosenthal A, Wild PJ. Molecular profiling of laser-microdissected matched tumor and normal breast tissue identifies karyopherin alpha2 as a potential novel prognostic marker in breast cancer. Clin Cancer Res 2006;12:3950-60.

19. Mortezavi A, Hermanns T, Seifert HH, Baumgartner MK, Provenzano M, Sulser T, Burger M, Montani M, Ikenberg K, Hofstadter F, Hartmann A, Jaggi R, Moch H, Kristiansen G, Wild PJ. KPNA2 expression is an independent adverse predictor of biochemical recurrence after radical prostatectomy. Clin Cancer Res 2011;17:1111-21.

20. Winnepenninckx V, Lazar V, Michiels S, Dessen P, Stas M, Alonso SR, Avril MF, Ortiz Romero PL, Robert T, Balacescu O, Eggermont AM, Lenoir G, Sarasin A, Tursz T, van den Oord JJ, Spatz A, Melanoma Group of the European Organization for R, Treatment of $\mathrm{C}$. Gene expression profiling of primary cutaneous melanoma and clinical outcome. J Natl Cancer Inst 2006;98:472-82.

21. Hegi ME, Diserens AC, Gorlia T, Hamou MF, de Tribolet N, Weller M, Kros JM, Hainfellner JA, Mason W, Mariani L, Bromberg JE, Hau P, Mirimanoff RO, Cairncross JG, Janzer RC, Stupp R. MGMT gene silencing and benefit from temozolomide in glioblastoma. N Engl $J$ Med 2005;352:997-1003.

22. Hartmann C, Hentschel B, Wick W, Capper D, Felsberg J, Simon M, Westphal M, Schackert G, Meyermann R, Pietsch T, Reifenberger G, Weller M, Loeffler M, von Deimling A. Patients with IDH1 wild type anaplastic astrocytomas exhibit worse prognosis than IDH1-mutated glioblastomas, and IDH1 mutation status accounts for the unfavorable prognostic effect of higher age: implications for classification of gliomas. Acta Neuropathol 2010;120:707-18.

23. Tabatabai G, Stupp R, van den Bent MJ, Hegi ME, Tonn JC, Wick W, Weller M. Molecular diagnostics of gliomas: the clinical perspective. Acta Neuropathol 2010;120:585-92.

24. Cross JL, Kott K, Miletic T, Johnson P. CD45 regulates TLR-induced proinflammatory cytokine and IFN-beta secretion in dendritic cells. J Immunol 2008;180:8020-9.

25. Almand B, Clark JI, Nikitina E, van Beynen J, English NR, Knight SC, Carbone DP, Gabrilovich DI. Increased production of immature myeloid cells in cancer patients: a mechanism of immunosuppression in cancer. J Immunol 2001;166:678-89.

26. O'Doherty U, Steinman RM, Peng M, Cameron PU, Gezelter S, Kopeloff I, Swiggard WJ, Pope M, Bhardwaj N. Dendritic cells freshly isolated from human blood express CD4 and mature into typical immunostimulatory dendritic cells after culture in monocyte-conditioned medium. J Exp Med 1993;178:1067-76.

27. Martín-Martín L, Almeida J, Hernández-Campo PM, Sánchez ML, Lécrevisse Q, Orfao A. Immunophenotypical, morphologic, and functional characterization of maturation-associated plasmacytoid dendritic cell subsets in normal adult human bone marrow. Transfusion 2009;49:1692-708.

28. Thomas R, Lipsky PE. Dendritic cells: origin and differentiation. Stem Cells 1996;14:196-206.

29. Mikeska T, Bock C, El-Maarri O, Hübner A, Ehrentraut D, Schramm J, Felsberg J, Kahl P, Buttner R, Pietsch T, Waha A. Optimization of quantitative MGMT promoter methylation analysis using pyrosequencing and combined bisulfite restriction analysis. J Mol Diagn 2007;9:368-81.

30. Pinzon-Charry A, Maxwell T, Prato S, Furnival C, Schmidt C, López JA. HLA-DR+immature cells exhibit reduced antigen-presenting cell function but respond to CD40 stimulation. Neoplasia 2005; 7:1123-32.

31. Lind EF, Ahonen CL, Wasiuk A, Kosaka Y, Becher B, Bennett KA, Noelle RJ. Dendritic cells require the NF-kappaB2 pathway for cross-presentation of soluble antigens. J Immunol 2008;181:354-63.

32. Fagerlund R, Melén K, Cao X, Julkunen I. NF-kappaB p52, RelB and c-Rel are transported into the nucleus via a subset of importin alpha molecules. Cell Signal 2008;20:1442-51.

33. Chemnitz J, Turza N, Hauber I, Steinkasserer A, Hauber J. The karyopherin CRM1 is required for dendritic cell maturation. Immunobiology 2010;215:370-9.

Cite this article as: Gousias K, von Ruecker A, Gielen GH, Niehusmann P, Waha A, Vatter H, Simon M. A potential role of karyopherin a2 in the impaired maturation of dendritic cells observed in glioblastoma patients. Neuroimmunol Neuroinflammation 2015;2(1):8-14.

Source of Support: Nil. Conflict of Interest: No.

Received: 20-08-2014; Accepted: 16-09-2014 